\title{
Local failure patterns for patients with nasopharyngeal carcinoma after intensity-modulated radiotherapy
}

\author{
Jia-xin Li ${ }^{1,2}$, Shao-min Huang ${ }^{1}$, Xin-hua Jiang ${ }^{3}$, Bin Ouyang ${ }^{2}$, Fei Han ${ }^{1}$, Shuai Liu', Bi-xiu Wen² and Tai-xiang Lu*
}

\begin{abstract}
Background: To investigate the clinical feature and the local failure patterns after intensity-modulated radiotherapy for nasopharyngeal carcinoma.

Methods: Between March 2007 and July 2009, 710 patients with nasopharyngeal carcinoma were treated with intensity-modulated radiotherapy. The magnetic resonance imagings obtained at recurrence were registered with the original planning computed tomography for dosimetry analysis.

Results: With a median follow-up of 38 months, 34 patients have developed local recurrence (32 cases valid). The incidence of invasion to nasopharynx, parapharyngeal space and the retropharyngeal space by the primary tumors was $100 \%, 75.0 \%$ and $62.5 \%$, respectively, but $78.1 \%, 34.4 \%$ and $21.9 \%$ at recurrence, respectively. The rate of invasion to ethmoid sinus was $3.1 \%$ by the primary tumors but $28.1 \%$ at recurrence $(p=0.005)$. The topographic analysis of the local failure patterns showed "central" in 16 patients; "marginal" in 9; and "outside" in 7. The median volumes of primary gross tumor were $45.84 \mathrm{~cm}^{3}$ in the central failure group, $29.44 \mathrm{~cm}^{3}$ in the marginal failure group, and $21.52 \mathrm{~cm}^{3}$ in the outside failure group, respectively $(p=0.012)$, and the median volumes of primary clinical target 1 were $87.28 \mathrm{~cm}^{3}, 61.90 \mathrm{~cm}^{3}$ and $58.74 \mathrm{~cm}^{3}$ in the three groups, respectively $(p=0.033)$.

Conclusions: In patients with nasopharyngeal carcinoma treated with intensity-modulated radiotherapy, the recurrent tumors had their unique characteristic and regularity of invasion to adjacent structures. "Central" failure was the major local failure pattern. The volumes of primary gross tumor and clinical target1 were significantly correlated with recurrent patterns. Employ more aggressive approaches to tumor cells which will be insensitive to radiotherapy may be an effective way to reduce the central failure.
\end{abstract}

Keywords: Nasopharyngeal carcinoma, Neoplasm recurrence, Local, Radiometry, Radiotherapy, Intensity-modulated, Treatment failure

\section{Background}

Nasopharyngeal carcinoma (NPC) has a distinct epidemiology compared with other head and neck squamous cell carcinomas. Standard treatment for NPC is radiotherapy (RT) for early-stage lesions or chemoradiotherapy for more advanced diseases. However, local-regional relapse after conventional RT remains one of the major treatment failures. In researches with optimized conventional RT and a more frequent use of chemotherapy in the 1990s,

\footnotetext{
* Correspondence: taixianglu@126.com

'Department of Radiation Oncology, Cancer Center, Sun Yat-Sen University, and State Key Laboratory of Oncology in Southern China, 651 Dongfeng Road East, Guangzhou 510060, P.R. China

Full list of author information is available at the end of the article
}

the 5-year local-regional failure rate of $15.0 \%-19.1 \%$ has been reported [1-3]. Studies have shown that the radiation dose delivered to the target volume correlates strongly with local disease control [4]. However, dose escalation by conventional technique is limited by the tolerance of adjacent critical organs.

Intensity-modulated radiotherapy (IMRT) is a major breakthrough in the treatment of NPC. By conforming the doses to the irregularly shaped tumor, dose escalation is possible with IMRT, which can potentially lead to improvement in local control [5,6]. Early treatment outcome was encouraging and conformed the promising role of IMRT [7]. Series with longer follow-up have also 
demonstrated excellent outcome: the 3-year locoregional control exceeded $90 \%$ in both early stage and advanced NPC $[8,9]$.

However, it is critical to examine the patterns of recurrence after IMRT, since one must consider the potential that: (1) the image-based target definitions used in IMRT might lead to smaller target volumes; (2) the conformal treatment plans and radiation delivery techniques that are used to minimize the dose to adjacent normal tissues might potentially increase the risk of marginal miss. The present study investigates the regularity of recurrent tumor invading to adjacent structures and make a detailed analysis to the dosimetric relationship between the patterns of failure and the initial irradiated dose of the recurrent site, which may provide information that is helpful in the future target volume definition and margin choices.

\section{Methods}

\section{Patient selection}

Between March 2007 and July 2009, 710 non-metastatic NPC patients were treated with IMRT at the Cancer Center of Sun Yat-sen University. The male/female ratio was nearly 3.2:1, and age ranged from 19 to 78 years (median, 45 years). All patients underwent disease staging according to the UICC 2009 staging system [10]. A total of 29 patients were in stage I; 92 in stage II; 390 in stage III; and 199 in stage IVa-b. T-classification was as follows: T1: 89; T2: 127; T3: 329; and T4: 165.

\section{Radiotherapy}

Patients were immobilized in the supine treatment position by a thermoplastic head and shoulder device. The CT images were obtained and transferred to the treatment-planning system, an inverse planning system (CORVUS 3.0/3.2, Peacock plan) developed by NOMOS Corporation.

Target volumes and normal tissues were delineated out on each slice. The gross tumor volume (GTV) included the primary nasopharyngeal tumor (GTVnx) and involved lymph nodes (GTVnd) as shown by clinical, endoscopic, and radiologic findings. For patients given induction chemotherapy, the targets were based on the post-chemotherapy extent as shown on the MRI images. Two clinical target volumes (CTVs) were delineated. CTV1 was defined as the GTVnx plus a 5-10 $\mathrm{mm}$ margin to encompass the high-risk sites of microscopic extension, including the entire nasopharynx mucosa plus a $5 \mathrm{~mm}$ submucosal volume. CTV2 was defined as the CTV1 plus a 5-10 mm margin (3-5 mm margin posteriorly) to encompass the low-risk sites of microscopic extension (including the parapharyngeal spaces, posterior third of nasal cavities and maxillary sinuses, pterygoid processes, pterygoid fossae, base of skull, lower half of sphenoid sinus, anterior half of clivus, and petrous apex), and lymphatic regions (the retropharyngeal lymph nodal regions, bilateral levels II, III, and Va were routinely covered in all patients, whereas ipsilateral levels IV, Vb, or supraclavicular fossae were also included for patients whose positive lymph nodes located lower than level II). Planning target volumes (PTVs) for all GTVs and CTVs were generated automatically according to the immobilization and localization uncertainties.

The prescribed dose was 68Gy to the PTVnx, 60Gy to the PTV1, 54Gy to the PTV2, and 64-66Gy to the PTVnd in 30 fractions. All patients were treated with one fraction daily over 5 days per week. The whole process of IMRT was carried out according to an institutional treatment protocol previously described [11]. The actual dose distributions for the treatment targets are listed in Table 1.

\section{Chemotherapy}

Our institutional guidelines recommended IMRT alone for patients in stage I-II, and IMRT combined with concurrent chemotherapy for those in stage III-IVb. Neoadjuvant cisplatin-based chemotherapy was applied in those patients with bulky neck node $(>3 \mathrm{~cm})$, and adjuvant chemotherapy was used for those patients with residual disease after IMRT.

\section{Follow-up and statistical analysis}

After IMRT completion, the patients were subsequently followed up monthly in the first 3 month, every 3 months through the first 3 years, then annually. During every follow-up visit, disease status and treatment toxicity were assessed. MRI was ordered on a regular basis and as clinically indicated. All recurrence scans were reviewed by the experienced radiologists and radiation oncologists. Patients who had complete response but developed recurrence at the primary site more than 6 months following completion of radical radiotherapy with or without chemotherapy were identified as having a recurrence. All analyses were performed in SPSS 16.0. All statistical tests were two-sided, and $p<0.05$ was considered as statistical significance.

\section{Dataset registration}

As has been described before [12,13], the MRI images obtained at the time when recurrence was diagnosed were input into the planning system, and each scan study became a dataset, which was a geometrically selfconsistent set of image data. The initial CT dataset served as the basis for all the registrations, meaning that the MRI datasets were moved so they were registered with the initial CT geometry. Bony, vascular, and muscular structures in proximity to the failure were used to guide the co-registration process. This process was continued until satisfactory visual agreement was obtained between the MRI surfaces and CT images. 
Table 1 Prescription dose to target volumes and dose-volume statistics

\begin{tabular}{ccccccc}
\hline Target & Goal (Gy) & $\begin{array}{c}\text { Median below } \\
\text { goal (\%) }\end{array}$ & $\begin{array}{c}\text { Median minimal } \\
\text { dose (Gy) }\end{array}$ & $\begin{array}{c}\text { Median maximal } \\
\text { dose (Gy) }\end{array}$ & $\begin{array}{c}\text { Median mean } \\
\text { dose (Gy) }\end{array}$ & Median volume (cm ${ }^{\mathbf{3}}$ ) \\
\hline GTVnx & 68 & 0.18 & 65.57 & 81.24 & 74.82 & 33.62 \\
CTV1 & 60 & 0.33 & 55.67 & 79.88 & 70.35 & 68.25 \\
CTV2 & 54 & 2.07 & 39.25 & 78.21 & 63.52 & 322.97 \\
GTVnd(L) & $60-64$ & 0.00 & 62.01 & 72.20 & 68.46 & 4.98 \\
GTVnd(R) & $60-64$ & 0.00 & 61.95 & 72.15 & 68.02 & 5.96 \\
\hline
\end{tabular}

Abbreviations: GTVnx primary nasopharyngeal gross tumor volume, CTV clinical target volume, GTVnd(L) GTV for the involved left cervical lymph nodes, GTVnd(R) GTV for the involved right cervical lymph nodes.

Dosimetric and target volume analysis of the recurrence The gross recurrent tumor volume (GTVr) was identified on MRI images while recurrence and transferred to the original planning CT. Further more, according to the relationship between GTVr and target volumes of the initial disease, TG (the intersection of GTVr and GTVnx), TC1 (the intersection of GTVr and CTV1, excluding TG), TC2 (the intersection of GTVr and CTV2, excluding TG and TC1) and TE (GTVr that outside the range of CTV2) were also delineated out (Figure 1), which represent recurrence in the center of the target, high-dose region, lowdose region and outside the target respectively.

Doses delivered to the recurrence volumes were calculated (Figure 2). Analysis of the recurrence patterns with respect to the dose distribution was initially performed by evaluating the median minimal dose, maximal dose and mean dose of GTVr, TG, TC1, TC2, TE, respectively. The percentages of the GTVr that TG, TC1, TC2 and TE accounted for were also calculated.

Three different categories were chosen for a sorting of local failure patterns:

Central: $\geq 50 \%$ of GTVr was within primary GTV (TG $\geq 50 \%$ of $\mathrm{GTVr}$ ).

Marginal: $\geq 50 \%$ of GTVr was inside primary CTV but outside primary GTV (TC1 + TC2 $\geq 50 \%$ of GTVr).
Outside: $\geq 50 \%$ of GTVr was outside primary CTV (TE $\geq 50 \%$ of $\mathrm{GTVr}$ ).

\section{Results}

Patient characteristics

After a median duration of 38 months (range, 659 months), 34 patients have developed local recurrence. In these patients, the male:female ratio was 3.6:1 and the median age was 41.5 years (range: $30-61$ years). The median duration from the end of the primary treatment to the diagnosis of recurrence was 24.0 months (range: 9.037.0 months). Twenty-five patients had biopsy-proven recurrence, all of which were WHO III. The remaining 9 were diagnosed based on progressive symptoms associated with new image findings that would explain the symptoms, of which 4 with MRI and 5 with MRI together with $\left[{ }^{18} \mathrm{~F}\right]$ fluorodeoxyglucose positron emission tomography scan. The usual condition was the patients with tumor over deep submucosa, basilar skull or intracranial area adjacent to the critical structures that might have technique difficulty or high morbidity probability for biopsy.

Among the 34 patients, the original IMRT plans of two patients were lost. Therefore, we concentrated our research on the remaining 32 patients. The median volume of GTVnx was $33.23 \mathrm{~cm}^{3}$, ranging from 6.08 to $91.43 \mathrm{~cm}^{3}$.

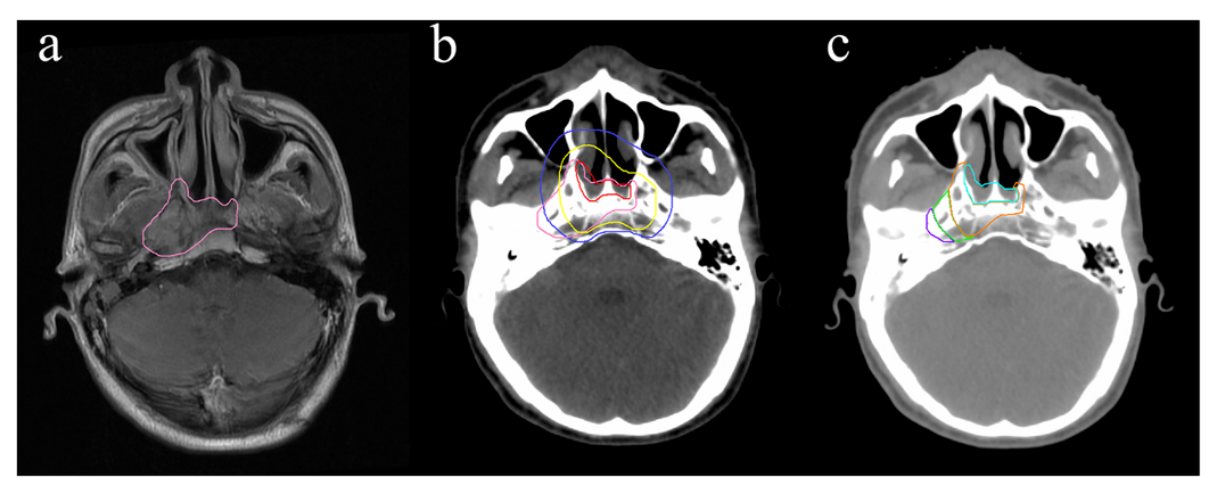

Figure 1 Delineation of target volumes. (a) The gross recurrent tumor volume (GTVr) was identified on MRI images. (b) The GTVr was transferred to the original planning CT. Red line is gross tumor volume (GTV); yellow line, CTV1; blue line, CTV2. (c) Cyan line, TG; orange line, TC1; green line, TC2; purple line, TE; TG+TC1+TC2+TE=GTVr. 


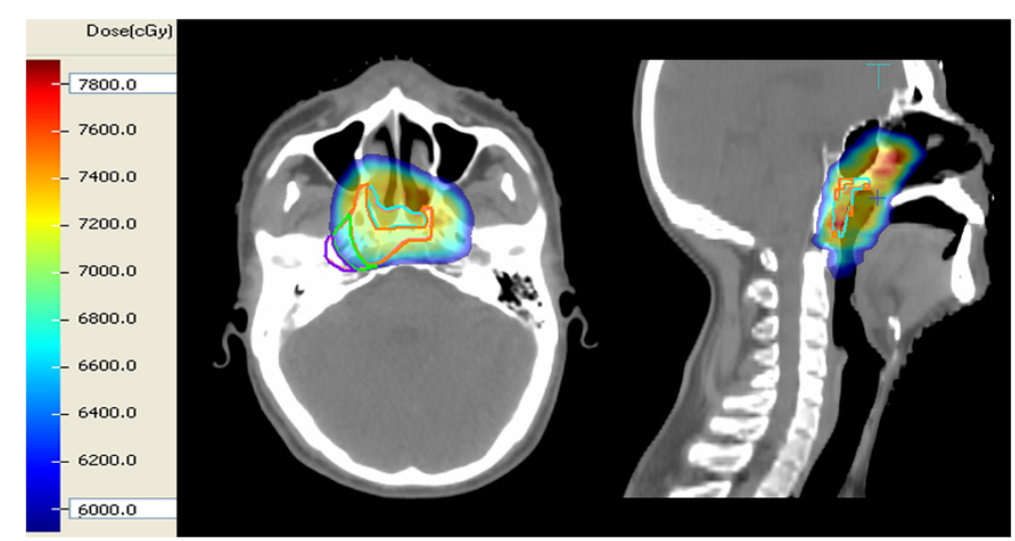

Figure 2 Dose delivered to the recurrent site in primary treatment.

The median mean doses of GTVnx, CTV1, and CTV2 were 74.82Gy, 70.50Gy and 63.52Gy, respectively.

The recurrent disease was Stage I in 4 (12.5\%), Stage II in 3 (9.4\%), Stage III in 11 (34.4\%), Stage IVa in 12 (37.5\%), Stage IVb in 1 (3.1\%), and Stage IVc in 1 (3.1\%) patients. A correlation between the T-stages at initial diagnosis and at recurrence (rT) was shown in Table 2. Twelve patients had the same T-stages at both diagnoses. Fifteen patients' recurrent T-stages were more advanced than the primaries', while 5 patients' primary T-stages were more advanced than the recurrence's $(p=0.064)$.

For further study, sites of primary and recurrent tumor invasion were compared by McNemar test (Table 3). The results showed that the invasion rates of the nasopharynx, parapharyngeal space and the retropharyngeal space by the recurrent tumor were significantly lower than those at primary. Conversely, the invasion rate of the ethmoid sinus was significantly higher at recurrence.

As the recurrent tumor volumes were contoured within the planning system, the median mean doses of GTVr, TG, TC1, TC2 and TE were 70.86Gy, 75.00Gy, 71.27Gy, 64.39Gy and 38.10Gy, respectively, and the median mean volumes of GTVr, TG, TC1, TC2 and TE were $29.33 \mathrm{~cm}^{3}, 8.74 \mathrm{~cm}^{3}, 7.08 \mathrm{~cm}^{3}, 2.88 \mathrm{~cm}^{3}$ and $0.38 \mathrm{~cm}^{3}$, respectively.

Table 2 Relationship between T classification at initial diagnosis and at recurrence

\begin{tabular}{cccccc}
\hline $\begin{array}{c}\text { Initial T } \\
\text { classification }\end{array}$ & \multicolumn{2}{c}{ No. patients by recurrent T classification } & Total \\
\cline { 2 - 5 } & $\mathbf{r T 1}$ & $\mathbf{r T 2}$ & $\mathbf{r T 3}$ & $\mathbf{r T 4}$ & \\
\hline T1 & 2 & 2 & 2 & 0 & 6 \\
T2 & 1 & 1 & 3 & 2 & 7 \\
T3 & 2 & 0 & 5 & 6 & 13 \\
T4 & 0 & 0 & 2 & 4 & 6 \\
Total & 5 & 3 & 12 & 12 & 32 \\
\hline
\end{tabular}

The topographic analysis of the local failure patterns showed "central" in 16 patients (50.0\%); "marginal" in 9 patients (28.1\%); and "outside" in 7 patient (21.9\%).

Factors that may influence the recurrence patterns were analyzed, including the histological type at initial diagnosis, stage of initial disease, dosimetric parameters of initial IMRT plan, residue or no while initial treatment finished and interval to recurrence. It revealed only 2 significant factors: the volume of GTVnx and CTV1. The median volumes of GTVnx were $45.84 \mathrm{~cm}^{3}$ in the central failure group, $29.44 \mathrm{~cm}^{3}$ in the marginal failure group, and $21.52 \mathrm{~cm}^{3}$ in the outside failure group, respectively $(p=0.012)$, and the median volumes of CTV1 were $87.28 \mathrm{~cm}^{3}, 61.90 \mathrm{~cm}^{3}$ and $58.74 \mathrm{~cm}^{3}$ in the three groups, respectively $(p=0.033)$.

\section{Discussion}

IMRT is a highly conformal radiation technique enabling delivery of high radiation doses to the gross tumor and high-risk areas while sparing the adjacent organs. Since the dose distribution is significant different between IMRT and conventional RT, is there also some unique quality with the failure patterns after IMRT? This study summarized the experience through the local failed cases in large number of patients with NPC, in hopes that the application of IMRT can be further optimized.

In our patients, the majority had more advanced T-stages at recurrence than that at initial diagnosis. Nasopharynx and structures adjacent to it, such as the parapharyngeal space and retropharyngeal space, the probability of invasion could be observed to be significantly lower at recurrence. Conversely, the probability of invasion to the ethmoid sinus was significantly higher at recurrence. As the definition of CTV required, the parapharyngeal space and retropharyngeal space are usually inside the CTV1. The mean dose of CTV1 at initial radiotherapy was about 70Gy in our patients, and the 
Table 3 Comparison of tumor invasion in patients with pNPC and rNPC

\begin{tabular}{|c|c|c|c|c|c|}
\hline Tumor invasion & $\begin{array}{c}\text { Invasion in pNPC and } \\
\text { rNPC (case) }\end{array}$ & $\begin{array}{l}\text { Invasion in pNPC } \\
\text { alone (case) }\end{array}$ & $\begin{array}{l}\text { Invasion in rNPC } \\
\text { alone (case) }\end{array}$ & $\begin{array}{c}\text { No invasion in pNPC } \\
\text { and rNPC (case) }\end{array}$ & $P$-value \\
\hline Nasopharynx & 25 & 7 & 0 & 0 & 0.008 \\
\hline Oropharynx & 3 & 3 & 2 & 24 & 0.655 \\
\hline Nasal cavity & 6 & 1 & 6 & 19 & 0.059 \\
\hline Laryngopharynx & 0 & 0 & 2 & 30 & 0.157 \\
\hline Soft palate & 0 & 0 & 3 & 29 & 0.083 \\
\hline Parapharyngeal space & 11 & 13 & 0 & 8 & $<0.001$ \\
\hline Longus capitis & 5 & 9 & 4 & 14 & 0.052 \\
\hline Retropharyngeal space & 6 & 14 & 1 & 11 & 0.001 \\
\hline Skull base & 15 & 4 & 4 & 9 & 1.000 \\
\hline Sphenoid sinus & 3 & 5 & 7 & 17 & 0.564 \\
\hline Ethmoid sinus & 1 & 0 & 8 & 23 & 0.005 \\
\hline Masticator space & 1 & 3 & 3 & 25 & 1.000 \\
\hline Cavernous sinus & 2 & 2 & 3 & 25 & 0.655 \\
\hline Intracranial & 1 & 0 & 2 & 29 & 0.157 \\
\hline
\end{tabular}

Abbreviations: $P N P C$ primary nasopharyngeal carcinoma, $r N P C$ recurrent nasopharyngeal carcinoma.

biological dose would be even higher with IMRT. We speculate such a high dosage would devastate the normal structure of nasopharynx, with the local blood supply reduced and unfavorable for tumor growth, and the tumor relocates and occurs far away from the nasopharynx.

The posterior ethmoid was included in the standard two-dimensional(2-D) radiation fields [14], even patients with $\mathrm{T} 1 / \mathrm{T} 2$ stages, so relapse in the ethmoid sinus was not common. However, IMRT can create a more conformal distribution around the targets, and the highdose region is defined by the precise three-dimensional (3-D) sectional anatomy. So the CTV should be smaller with IMRT. Physicians initially attempted to use IMRT by creating CTV on the basis of past experiences with 2$\mathrm{D}$ planning, the law of local invasion and the radiological information. There is no appreciable differences with various definitions of CTV $[11,15,16]$, and the rates of locoregional control were similar and satisfied. But the vast majority of these definitions did not mention if the posterior ethmoid sinus should be included in the CTV.

The ethmoid sinus is the earliest developed of the nasal sinus, and it tends to spread as far as there is any compact bone, which leads to great variability with the anatomical position of the posterior border of the ethmoid sinus. It was reported that $66 \%$ of the posterior ethmoid sinus intruded into the sphenoid body, $8 \%$ intruded into the lesser wing of sphenoid bone, $38 \%$ spreaded downward and resulted in middle turbinate gasification [17]. Thus, the ethmoid sinus has a close relation with the surrounding anatomic structures. A larger sample study is expected to identify whether the posterior ethmoid sinus should be included in the high-risk area.

In the local failure patterns after 3D-RT, a study retrospectively reviewed 151 patients with head-and-neck cancer(HNC), 14 of whom developed local recurrences, which showed 12 were in-field failure, 1 was marginal, and 1 was out-of-field [18]. Since IMRT became rapidly utilized in the treatment of HNC in the late 1990s, the recurrent patterns of IMRT have been studied in limited published literature. Chao et al. analyzed $126 \mathrm{HNC}$ patients treated with IMRT delivered with radical intent without surgery in $41 \%$ of patients, and post-operatively in 59\% of patients [19]. After a median follow up of 26 months, 17 recurrences were noted, of which 9 were in-field, 3 were marginal failures and 5 were outside of the IMRT field. Studer et al. reported $280 \mathrm{HNC}$ patients treated with IMRT, in $75 \%$ of whom definitive IMRT was performed [20]. After a mean follow-up of 23.2 months, 46 local failures have been observed, 45 of which were confirmed "in field". $\mathrm{Ng}$ et al. reported the treatment outcomes of 193 NPC patients with a median follow up of 30 months [21]. There were 16 local failures, 13 of that were considered in field.

In our series, local failure patterns were categorized according to the relationship of recurrent tumors and primary target volumes, which could evaluate the rationality of target volume delineation in our institution more effectively. The result demonstrated that approximately $80 \%$ of the recurrent tumors were located mainly inside CTV, with the median mean dose of GTVr as 70.86Gy. It seems that the delineation of target volumes are reasonable, but why failures in the region originally 
assigned to the "full dose" needs further discussion. In analysis of factors that may influence the recurrence patterns, it was found that the median volume of GTVnx and CTV1 in the group "central failure" were significantly larger than those in the other 2 groups. It is concluded that the large size tumors might not be really cured under the conventional radical dose. Shen et al. reported 154 patients with NPC treated with accelerated hyperfractionated radiotherapy [22]. The 5-year local failure-free rate were $89.4 \%$ vs $48.9 \%(p=0.002)$, respectively, for patients whose GTV-P were $\leq 60 \mathrm{ml}$ and $>60 \mathrm{ml}$. In a study with 290 NPC patients [23], PTV $>60$ cc were associated with significantly poorer local control $(p<0.001)$. In the early 1980s, Fletcher has proposed that a certain tumor volume requires a certain radiation dose so as to get a radical cure. Tumors about $3 \mathrm{~cm}^{3}$ should not be irradiated less than 75Gy, and the larger ones even need more than 100Gy [24]. Therefore, larger tumors require higher doses for their control. We should design a more individual treatment plan to the large tumors, raising the prescription dose in hopes of "real cure".

A previous study showed that tumors less than $1 \mathrm{~mm}$ in diameter were well-oxygenated, and hypoxia would occur when the tumors grew larger [25]. Hypoxic mammalian cells are 2.5 to 3 times less radiosensitive than well-oxygenated cells. In vitro and in vivo measurements have shown that an increased radiation dose may overcome hypoxic resistance [26]. However, increasing the radiation dose indiscriminately may increase normal tissue complication rates. One approach is to implement hypoxia imaging-guided IMRT for dose escalation. So far, there were experimental application with ${ }^{18} \mathrm{~F}$-FMI$\mathrm{SO},{ }^{18} \mathrm{~F}$-FAZA and ${ }^{62} \mathrm{Cu}$-ATSM PET to guide IMRT planning on a small scale [27-29]. Another potential approach is to develop oxygen-mimetic, electron-affinic radiosensitizers. These radiosensitizers are easier to administer than oxygen, less rapidly metabolized, and can therefore diffuse further from blood vessels into the hypoxic regions of the tumor. Toxicity has somewhat limited their use, but there was evidence of clinical efficacy $[30,31]$.

In present study, there were 9 "marginal failure" and 7 "outside the field", all of which the primary tumor extents and initial IMRT plans were reappraised. It was found that defects in target volume delineation in 4 cases. Among them, 2 primary tumors had invaded to the oropharynx and parapharyngeal space, but they were not completely included in GTV, which led to relapse in oropharynx and laryngopharynx (Figure 3). The other two patients with clivus or sphenoid bone invaded at initial diagnosis, but they were not well included in GTV, which led to recurrences in skull base and the ethmoid sinus. The high conformity and steep dose gradients

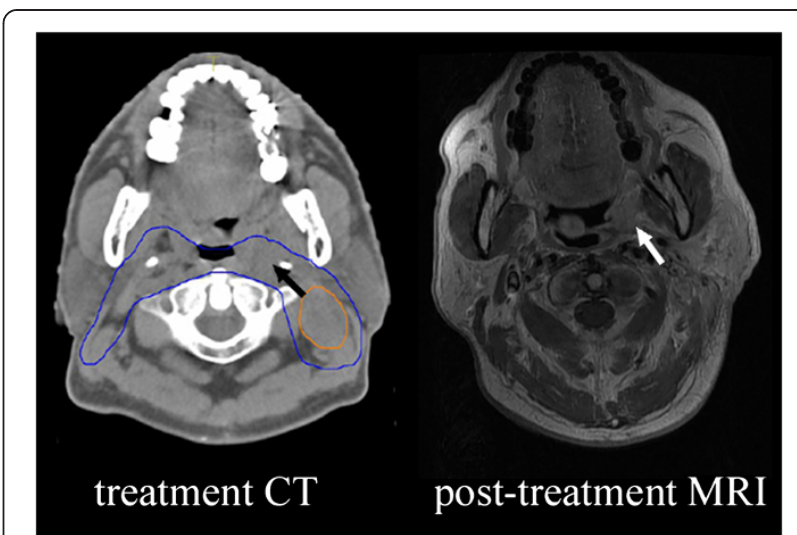

Figure 3 One patient with marginal failure. Part of primary tumor was just inside CTV2 (blue line), but outside GTV or CTV1. The white arrow indicates the recurrent tumor.

with IMRT have made the accurate target volume delineation of outmost importance. Any fault or carelessness in delineation can result in overdose of the critical structure or escape of the target volume. Some measures can be adopted to improve the accuracy of target volume delineation, including establishment of guidelines for target volume delineation, obtaining high-quality image (using image fusion technology if necessary) and organizing consultations with radiologists.

There is no physiological activity like peristalsis or aspiratory movement that can cause significant anatomic changes in the nasopharynx nearby, but shrinking of the primary tumor or nodal masses, body weight loss and set-up error during fractionated RT may lead to changes in body contour, target volumes and risk organs. They would affect the dose distribution and can be the root of marginal recurrence. It was reported that patients with NPC or with great weight loss or reduction in neck separation did have clinically significant benefits with adaptive RT using helical tomotherapy [32]. We hope the benefits would eventually bring improvement in local control rate.

\section{Conclusions}

Our study investigated the local failure patterns of NPC patients treated with IMRT in a large cohort. Based on our results, the recurrent tumors had their unique character and regularity of invasion to adjacent structures. "Central" failure was the major local failure pattern. The volumes of GTVnx and CTV1 were significantly correlated with recurrent patterns. Identify the tumor cells which will be insensitive to RT and employ more aggressive and target-specific therapeutic approaches may be an effective way to reduce the central failure. 


\section{Consent}

Written informed consent was obtained from the patient for the publication of this report and any accompanying images.

\section{Competing interests}

The authors declare that they have no competing interests.

\section{Authors' contributions}

JXL carried out data collection, data analysis, manuscript drafting and revision. SMH, BO and SL contributed to the dosimetric data analysis and interpretation. XHJ aided in the image data collection. FH and BXW contributed in writing manuscript. TXL initially developed the concept of the study and contributed in writing manuscript and all revisions. All authors read and approved the final manuscript.

\section{Author details}

'Department of Radiation Oncology, Cancer Center, Sun Yat-Sen University, and State Key Laboratory of Oncology in Southern China, 651 Dongfeng Road East, Guangzhou 510060, P.R. China. ${ }^{2}$ Department of Radiation Oncology, The First Affiliated Hospital, Sun Yat-Sen University, 58 Zhongshan Road II, Guangzhou 510080, P.R. China. ${ }^{3}$ Imaging Diagnosis and Interventional Center, Cancer Center, Sun Yat-Sen University, and State Key Laboratory of Oncology in Southern China, 651 Dongfeng Road East, Guangzhou 510060, P.R. China.

Received: 15 November 2013 Accepted: 9 March 2014 Published: 27 March 2014

\section{References}

1. Lee AW, Sez WM, Au JS, Leung SF, Leung TW, Chua DT, Zee BC, Law SC, Teo PM, Tung SY, Kwong DL, Lau WH: Treatment results for nasopharyngeal carcinoma in the modern era: the Hongkong experience. Int I Radiat Oncol Biol Phys 2005, 61:1107-1116.

2. Leung TW, Tung SY, Sze WK, Wong FC, Yuen KK, Lui CM, Lo SH, Ng TY, Saiki $\mathrm{O}$ : Treatment results of 1070 patients with nasopharyngeal carcinoma: an analysis of survival and failure patterns. Head Neck 2005, 27:555-565.

3. Yi JL, Gao L, Huan XD, Li SY, Luo JW, Cai WM, Xiao JP, Xu GZ: Nasopharyngeal carcinoma treated by radical radiotherapy alone: ten-year experience of a single institution. Int I Radiat Oncol Biol Phys 2006, 65:161-168.

4. Teo PM, Leung SF, Tung SY, Zee B, Sham JS, Lee AW, Lau WH, Kwan WH, Leung TW, Chua D, Sze WM, Au JS, Yu KH, Saiki O, Kwong D, Yau TK, Law SC, Sze WK, Au G, Chan AT: Dose-response relationship of nasopharyngeal carcinoma above conventional tumoricidal level: a study by the Hong Kong Nasopharyngeal Carcinoma Study Group (HKNPCSG). Radiother Oncol 2006, 79:27-33.

5. Xia P, Fu KK, Wong GW, Akazawa C, Verhey L: Comparison of treatment plans involving intensity-modulated radiotherapy for nasopharyngeal carcinoma. Int J Radiat Oncol Biol Phys 2000, 48:329-337.

6. Kam MK, Chau RM, Suen J, Choi PH, Teo PM: Intensity-modulated radiotherapy in nasopharyngeal carcinoma: dosimetric advantage over conventional plans and feasibility of dose escalation. Int I Radiat Oncol Biol Phys 2003, 56:145-157.

7. Lee N, Xia P, Quivey JM, Sultanem K, Poon I, Akazawa C, Akazawa P, Weinberg V, Fu KK: Intensity-modulated radiotherapy in the treatment of nasopharyngeal carcinoma: an update of the UCSF experience. Int $J$ Radiat Oncol Biol Phys 2002, 53:12-22.

8. Lin S, Pan J, Han L, Zhang X, Liao X, Lu JJ: Nasopharyngeal carcinoma treated with reduced-volume intensity-modulated radiation therapy: report on the 3-year outcome of a prospective series. Int I Radiat Oncol Biol Phys 2009, 75:1071-1078.

9. Tham IW, Hee SW, Yeo RM, Salleh PB, Lee J, Tan TW, Fong KW, Chua ET, Wee $J$ T: Treatment of nasopharyngeal carcinoma using intensity-modulated radiotherapy-the national cancer centre singapore experience. Int J Radiat Oncol Biol Phys 2009, 75:1481-1486.

10. Sobin LH, Gospodaroeics MK, Wittekind C: TNM Classification of malignant tumours. 7th edition. New York: Wiley Press; 2009.

11. Xiao WW, Huang SM, Han F, Wu SX, Lu LX, Lin CG, Deng XW, Lu TX, Cui NJ, Zhao C: Local control, survival, and late toxicities of locally advanced nasopharyngeal carcinoma treated by simultaneous modulated accelerated radiotherapy combined with cisplatin concurrent chemotherapy: long-term results of a phase 2 study. Cancer 2011, 117:1874-1883.

12. Kessler ML, Pitluck S, Petti PL, Castro JR: Integration of multimodality imaging data for radiotherapy treatment planning. Int J Radiat Oncol Biol Phys 1991, 21:1653-1667.

13. Ten Haken RK, Thornton AF, Sandler HM, LaVigne ML, Quint DJ, Fraass BA, Kessler ML, McShan DL: A quantitative assessment of the addition of MRI to CT-based, 3-D treatment planning of brain tumors. Radiother Oncol 1992, 25:121-133.

14. Chao KS, Perez CA: Nasopharynx. In Principles and practice of radiation oncology. 4th edition. Edited by Perez C, Brady L, Halperin E, Schmidt-Ullrich R. Philadelphia: Lippincott Williams \& Wilkins; 2003:930.

15. Lee N, Harris J, Garden AS, Straube W, Glisson B, Xia P, Bosch W, Morrison WH, Quivey J, Thorstad W, Jones C, Ang KK: Intensity-modulated radiation therapy with or without chemotherapy for nasopharyngeal carcinoma: radiation therapy oncology group phase II trial 0225. J Clin Oncol 2009, 27:3684-3690

16. Lee NY, Zhang Q, Pfister DG, Kim J, Garden AS, Mechalakos J, Hu K, Le QT, Colevas AD, Glisson BS, Chan AT, Ang KK: Addition of bevacizumab to standard chemoradiation for locoregionally advanced nasopharyngeal carcinoma (RTOG 0615): a phase 2 multi-institutional trial. Lancet Oncol 2012, 13:172-180.

17. Levine $H L$ : Functional endoscopic sinus surgery: evaluation surgery and follow-up of 250 patients. Laryngoscope 1990, 100:79-84

18. Oksuz DC, Prestwich RJ, Carey B, Wilson S, Senocak MS, Choudhury A, Dyker K, Coyle C, Sen M: Recurrence patterns of locally advanced head and neck squamous cell carcinoma after 3D conformal (chemo)-radiotherapy. Radiat Oncol 2011, 6:54.

19. Chao KS, Ozyigit G, Tran BN, Cengiz M, Dempsey JF, Low DA: Patterns of failure in patients receiving definitive and postoperative IMRT for head-and-neck cancer. Int J Radiat Oncol Biol Phys 2003, 55:312-321.

20. Studer G, Luetolf UM, Glanzmann C: Locoregional failure analysis in head-and-neck cancer patients treated with IMRT. Strahlenther Oncol 2007, 183:417-423.

21. Ng WT, Lee MC, Hung WM, Choi CW, Lee KC, Chan OS, Lee AW: Clinical outcomes and patterns of failure after intensity-modulated radiotherapy for nasopharyngeal carcinoma. Int J Radiat Oncol Biol Phys 2011, 79:420-428.

22. Shen C, Lu JJ, Gu Y, Zhu G, Hu C, He S: Prognostic impact of primary tumor volume in patients with nasopharyngeal carcinoma treated by definitive radiation therapy. Laryngoscope 2008, 118:1206-1210.

23. Chua DT, Sham JS, Kwong DL, Tai KS, Wu PM, Lo M, Yung A, Choy D, Leong $\mathrm{L}$ : Volumetric analysis of tumor extent in nasopharyngeal carcinoma and correlation with treatment outcome. Int J Radiat Oncol Biol Phys 1997, 39:71|-719.

24. Fletcher GH, Million RR: Nasopharynx. In Textbook of radiotherapy. 3rd edition. Edited by Fletcher GH. Philadelphia: Lea \& Febiger Press; 1980:364-383.

25. Stanley JA, Shipley WU, Steel GG: Influence of tumor size on hyopxic fraction and therapeutic sensitivity of Lewis lung tumor. Br J Cancer 1977, 36:105-113.

26. Hall EJ: Radiobiology for the radiologist. Philadelphia: JB Lippincott Press; 1994

27. Lee NY, Mechalakos JG, Nehmeh S, Lin Z, Squire OD, Cai S, Chan K, Zanzonico PB, Greco C, Ling CC, Humm JL, Schöder H: Fluorine-18-labeled fluoromisonidazole positron emission and computed tomographyguided intensity-modulated radiotherapy for head and neck cancer: a feasibility study. Int J Radiat Oncol Biol Phys 2008, 70:2-13.

28. Grosu AL, Souvatzoglou M, Röper B, Dobritz M, Wiedenmann N, Jacob V, Wester HJ, Reischl G, Machulla HJ, Schwaiger M, Molls M, Piert M: Hypoxia imaging with FAZA-PET and theoretical considerations with regard to dose painting for individualization of radiotherapy in patients with head and neck cancer. Int J Radiat Oncol Biol Phys 2007, 69:541-551.

29. Wong TZ, Lacy JL, Petry NA, Hawk TC, Sporn TA, Dewhirst MW, Vlahovic G: PET of hypoxia and perfusion with ${ }^{62} \mathrm{Cu}-\mathrm{ATSM}$ and ${ }^{62} \mathrm{Cu}$-PTSM using a ${ }^{62} \mathrm{Zn} /{ }^{62} \mathrm{Cu}$ generator. AJR Am J Roentgenol 2008, 190:427-432.

30. Overgaard J: Clinical evaluation of nitromidazoles as modifiers of hypoxia in solid tumors. Oncol Res 1994, 6:509-518.

31. Overgaard J, Hansen HS, Overgaard M, Bastholt L, Berthelsen A, Specht L, Lindeløv $B$, Jørgensen $K$ : A randomized double-blind phase III study of nimorazole as a hypoxic radiosensitizer of primary radiotherapy in 
supraglottic larynx and pharynx carcinoma. Results of the Danish Head and Neck Cancer Study (DAHANCA) Protocol 5-85. Radiother Oncol 1998, 46:135-146.

32. Capelle L, Mackenzie M, Field C, Parliament M, Ghosh S, Scrimger R: Adaptive radiotherapy using helical tomotherapy for head and neck cancer in definitive and postoperative settings: initial results. Clin Oncol (R Coll Radiol) 2012, 24:208-215.

doi:10.1186/1748-717X-9-87

Cite this article as: Li et al: Local failure patterns for patients with nasopharyngeal carcinoma after intensity-modulated radiotherapy. Radiation Oncology 2014 9:87.

\section{Submit your next manuscript to BioMed Central and take full advantage of:}

- Convenient online submission

- Thorough peer review

- No space constraints or color figure charges

- Immediate publication on acceptance

- Inclusion in PubMed, CAS, Scopus and Google Scholar

- Research which is freely available for redistribution 